\title{
Awareness of Doctors' Shared Decision-Making in Life-Sustaining Care Decisions
}

\section{Dalyong Kim, M.D., Hyun Jung Lee, Ph.D., Soo-Young Yu, Ph.D., M.P.H.*, Jung Hye Kwon, Ph.D. ${ }^{\dagger}$, Hee Kyung Ahn, Ph.D. ${ }^{\dagger}$, Jee Hyun Kim, Ph.D. ${ }^{\S}$, Seyoung Seo, Ph.D. ", Chi Hoon Maeng, Ph.D. ", Seungtaek Lim, M.D.**, Do Yeun Kim, Ph.D. and Sung Joon Shin, Ph.D. ${ }^{+\dagger}$}

Division of Hematology \& Medical Oncology, Department of Internal Medicine, Dongguk University llsan Hospital,

Dongguk University College of Medicine, Goyang, *Department of Nursing Science, Jeonju University, Jeonju,

${ }^{\dagger}$ Division of Hematology and Oncology, Department of Internal Medicine, Chungnam National University Sejong Hospital, Chungnam National University College of Medicine, Sejong, ${ }^{\ddagger}$ Division of Medical Oncology, Department of Internal Medicine, Gachon University Gil Medical Center, Incheon,

${ }^{\S}$ Division of Hematology/Medical Oncology, Department of Internal Medicine, Seoul National University Bundang Hospital, Seoul National University College of Medicine, Seongnam, "Department of Oncology,

University of Ulsan College of Medicine, Asan Medical Center, Seoul, "Division of Medical Oncology-Hematology, Department of Internal Medicine, Kyung Hee University Hospital, Kyung Hee University College of Medicine, Seoul, **Department of Hemato-Oncology, Wonju Severance Christian Hospital, Yonsei University Wonju College of Medicine, Wonju, ${ }^{\dagger \dagger}$ Division of Nephrology, Department of Internal Medicine, Dongguk University llsan Hospital, Dongguk University College of Medicine, Goyang, Korea

Purpose: At the end of life, communication is a key factor for good care. However, in clinical practice, it is difficult to adequately discuss end-of-life care. In order to understand and analyze how decision-making related to life-sustaining treatment (LST) is performed, the shared decision-making (SDM) behaviors of physicians were investigated. Methods: A questionnaire was designed after reviewing the literature on attitudes toward SDM or decision-making related to LST. A final item was added after consulting experts. The survey was completed by internal medicine residents and hematologists/medical oncologists who treat terminal cancer patients. Results: In total, 202 respondents completed the questionnaire, and $88.6 \%$ said that the decision to continue or end LST is usually a result of SDM since they believed that sufficient explanation is provided to patients and caregivers, patients and caregivers make their own decisions according to their values, and there is sufficient time for patients and caregivers to make a decision. Expected satisfaction with the decisionmaking process was the highest for caregivers (57.4\%), followed by physicians (49.5\%) and patients (41.1\%). In total, $38.1 \%$ of respondents said that SDM was adequately practiced when making decisions related to LST. The most common reason for inadequate SDM was time pressure (89.6\%). Conclusion: Although most physicians answered that they practiced SDM when making decisions regarding LST, satisfactory SDM is rarely practiced in the clinical field. A model for the proper implementation of SDM is needed, and additional studies must be conducted to develop an SDM model in collaboration with other academic organizations.

Key Words: Shared decision making, Advance care planning, Neoplasms
Received April 11, 2021

Revised October 2, 2021

Accepted October 4, 2021
Correspondence to

Do Yeun Kim

ORCID:

https://orcid.org/0000-0001-7265-1994

E-mail:smdkdy@duih.org

Sung Joon Shin

ORCID:

https://orcid.org/0000-0002-0777-9278

E-mail:shine5050@naver.com

This study was conducted with the support of the National Evidence-based Healthcare Collaborating Agency (NA19-008). 


\section{INTRODUCTION}

Communication is a key factor for high-quality care at the end of life [1]. During this time, doctors should inform patients and their caregivers about the severity of the illness and make decisions about treatment methods after providing evidencebased information and taking into consideration any particular values and wishes of the patient. Such interactive communication is referred to as shared-decision making [2], a concept that involves doctors fulfilling their obligation to provide adequate information and patients voicing their preference in making treatment decisions based on the ethical principle of autonomy. Doctor-patient shared-decision making can eliminate any obscurity through the process of confirming patients' wishes and preferences when facing decisions about various treatments that can be aggressive, expensive, and potentially ineffective during end-of-life care [3].

The Act on Hospice and Palliative Care and Decisions on Life-Sustaining Treatment for Patients at the End of Life (hereinafter referred to as the Act on Decisions on Life-Sustaining Treatment) was passed on February 3, 2016, and went into effect in February 2018. The basic principles of the act are as follows: "First, all activities concerning hospice care, lifesustaining treatment, and determination to terminate, etc., lifesustaining treatment shall not infringe on the human dignity and value of patients. Second, every patient has the right to receive the best treatment and clearly know about the status and prognosis of the injury or disease he/she suffers and the subsequent medical services, and to make decisions for himself/ herself thereon. Third, each medical person under the Medical Service Act shall provide patients with the best treatment, $\mathrm{ex}^{-}$ plain hospice, palliative care, and determination to terminate, etc., life-sustaining treatment, accurately and in detail, and shall respect the patient's decision made based thereon" [4].

The law emphasizes the importance of communication with patients and caretakers to make well-informed decisions. However, there is a gap between the basic principles and intentions of the law and its application in the clinical field. In a recent study of 132 clinicians at Seoul National University Hospital who make decisions regarding life-sustaining treatment, $86.4 \%$ reported serious difficulty discussing decisions about life-sustaining treatment [5]. Researchers in other countries with similar laws have suggested that the legal process of coordinating to advance directives or the withdrawal of life-sustaining treatment should be replaced with communication that respects the wishes of the patient [6].

Due to the enactment of the Act on Decisions on LifeSustaining Treatment in South Korea, it is necessary to understand not just the number of decisions related to lifesustaining treatment that need to be made but also the quality of the decision-making process. Such research can provide data for designing education programs for doctors who have to make decisions on life-sustaining treatment in the clinical field and improving relevant academic and government institutions. Most studies that have been used as a reference for doctors' perceptions related to shared decision-making about life-sustaining treatment have been conducted internationally, and no studies have been conducted that reflect the clinical environment and culture of South Korea. It has been observed that, even in other countries where discussions about patients' autonomous decision-making and the concept of shared decision-making prompt earlier discussions between patients and doctors when making decisions about end-of-life care, explicit conversations about the termination of treatment are rare [7]. In a study about shared decision-making during clinical practice with 351 oncologists, surgical oncologists, and radiation oncologists, $82 \%$ of participants responded that they did not receive relevant education, and $66 \%$ reported that they did not exercise shared decision-making in actual clinical practice [8].

In the Korean Professional Consensus for Comfort Care and Withdrawing/Withholding in the Intensive Care Unit published by the Korean Society of Critical Care Medicine in February 2018, sufficient family consulting and communication were emphasized as the core aspects of a dignified death in the process of removing a patient's ventilator and discontinuing extracorporeal membrane oxygenation. In particular, attending medical personnel should sufficiently discuss plans to withdraw or withhold life-sustaining treatment with patients and their family members, confirm that they understand the decision in full, provide information to them about the process, share their plans for the process of ending life-sustaining treatment, and proceed accordingly using clear communication methods [9]. In a 2012 study, Jo [10] evaluated the definition 
of shared decision-making used in South Korean society and developed a measurement scale for decision-making. Although Lee et al. [11] examined the perceptions of hospice and shared decision-making among healthy middle-aged adults, very few studies have examined the perspectives of doctors who perform the important role of facilitating decision-making about life-sustaining treatment. Recently, the Korean Society of Nephrology began a research project about shared decisionmaking related to the start time of dialysis and the choice of dialysis method for patients with end-stage renal failure [12]. Results from their study related to decision-making surrounding maintaining or withdrawing dialysis when patients become terminal or reach the end of life according to the Act on Decisions on Life-Sustaining Treatment are expected in the future and, together with this study, will suggest future directions related to shared decision-making.

This study aimed first to explore the self-evaluation, satisfaction, and expected satisfaction of patients and caregivers regarding shared decision-making related to life-sustaining treatment and the factors that influenced these variables. Second, the reasons participants believed their experiences making decisions about life-sustaining treatment were the result of shared decision-making were explored using a word cloud. Third, data were gathered that to support the development of a better doctor-patient decision-making model for those who make decisions about life-sustaining treatment, including hematologists/medical oncologists as well as internal medicine residents.

\section{METHODS}

\section{Selection of survey items}

Literature about shared decision-making and decisionmaking by doctors related to life-sustaining treatment was reviewed, and items were extracted based on the findings [13-16]. The first draft of the survey items was reviewed by 1 expert each from the Korean Society for Hospice and Palliative Care, the Korean Society for Medical Ethics, the Korean Association for Medical Law, and the Korean Academy on Communication in Healthcare before the survey items were finalized. The survey items included demographic information, shared decision-making in routine medical practice, shared decision-making in the process of making decisions about life-sustaining treatment, satisfaction about the decisionmaking process, and the appropriateness of the shared decision-making process.

\section{Ethical considerations}

This study was conducted after receiving approval from the internal review boards of each institution with the support of the National Evidence-based Healthcare Collaborating Agency (National Evidence-based Healthcare Collaborating Agency: NECAIRB19-005-7, Hallym University Sacred Heart Hospital: KANGDONG 2019-03-001-001, Gachon University Gil Medical Center: GBIRB2019-155, Seoul National University Bundang Hospital: B-1905/541-302, Seoul Asan Medical Center: S2019-1012-0002, Kyung Hee University Hospital: KHUH 2019-06-007-002, Yonsei University Wonju Severance Christian Hospital: CR319043, Dongguk University Ilsan Hospital: DUIH 2019-04-002-003). Based on the principles of the Helsinki Declaration (2008 revision following the 59th World Medical Association General Assembly in Seoul), this study was conducted both scientifically and ethically.

\section{Participants}

After confirming the intention to voluntarily participate in the study among hematologists/medical oncologists who were members of the ethics sub-committee of the Korean Cancer Study Group (KCSG) palliative medicine committee, a total of 7 hematologists/medical oncologists participated as representatives of their hospitals. The researcher assigned to each institution was responsible for securing internal review board approval, administering the survey, gathering the data, and conducting any other study activities for the corresponding institution. The researcher responsible for each institution verified the study participation of hematologists/medical oncologists and internal medicine residents, all of whom voluntarily consented to participate prior to the study.

\section{Data analysis}

Using SPSS version 25 (IBM Corp., Armonk, NY, USA), descriptive statistics were used to identify frequencies and proportions, and the chi-square test was conducted to identify 
group differences in the survey items. Participants with missing values for an item were excluded from the analysis of the corresponding item. Responses to the open-ended items were visualized using word clouds that extracted the most frequently used words. The word clouds were generated using the trial version of MAXQDA (VERBI Software, Berlin, Germany).

\section{RESULTS}

\section{General characteristics of participants}

The survey was distributed to 287 doctors across 7 hospitals in South Korea, and 202 (70.38\%) responded (Table 1).

Table 1. Baseline Characteristics ( $N=202)$.

\begin{tabular}{|c|c|}
\hline Variables & $\mathrm{n}(\%)$ \\
\hline \multicolumn{2}{|l|}{ Gender } \\
\hline Male & $102(50.5)$ \\
\hline Female & $100(49.5)$ \\
\hline \multicolumn{2}{|l|}{ Age (yr) } \\
\hline$<30$ & $37(18.3)$ \\
\hline 30 39 & $142(70.3)$ \\
\hline $40 \sim 49$ & $14(6.9)$ \\
\hline $50 \sim 59$ & $8(4.0)$ \\
\hline $60 \sim 65$ & $1(0.5)$ \\
\hline \multicolumn{2}{|l|}{ Position } \\
\hline Internal medicine resident & $159(78.7)$ \\
\hline Junior resident & $69(34.2)$ \\
\hline Senior resident & $90(44.6)$ \\
\hline Hematologist/medical oncologist & $43(21.3)$ \\
\hline Fellow & $11(5.4)$ \\
\hline Staff & $32(15.8)$ \\
\hline \multicolumn{2}{|l|}{ Type of institution } \\
\hline Tertiary referral hospital & $153(75.7)$ \\
\hline General hospital & $49(24.3)$ \\
\hline \multicolumn{2}{|l|}{ Average number of patients per week } \\
\hline$<20$ & $33(16.3)$ \\
\hline $20 \sim 49$ & 76 (37.6) \\
\hline $50 \sim 79$ & $42(20.8)$ \\
\hline 80 99 & $9(4.5)$ \\
\hline$\geq 100$ & $42(20.8)$ \\
\hline \multicolumn{2}{|c|}{$\begin{array}{l}\text { Number of patients who decided to suspend or } \\
\text { stop life-sustaining treatment in the past week }\end{array}$} \\
\hline$<2$ & $118(58.4)$ \\
\hline $2 \sim 4$ & $69(34.2)$ \\
\hline $5 \sim 6$ & $11(5.4)$ \\
\hline $7 \sim 9$ & $1(0.5)$ \\
\hline$\geq 10$ & $3(1.5)$ \\
\hline
\end{tabular}

In total, $50.5 \%$ of respondents were men, and $49.5 \%$ were women. Furthermore, $70.3 \%$ of respondents were in their 30s, and $75.5 \%$ of the participants worked at a tertiary hospital. A majority of the participants (78.7\%) were residents, and $21.3 \%$ were specialists. Seventy-six participants (37.6\%) answered that they treated 20 to 49 patients per week, followed by 42 (20.8\%) who treated 50 to 79 patients per week. Forty-two participants $(20.8 \%)$ responded that they treated more than 100 patients in the past week. The most frequent response to the item about the number of patients for whom the respondents made decisions about withholding or withdrawing lifesustaining treatment in the past week was less than $2(n=118$, $58 \%)$.

\section{Shared decision-making during routine medical practice}

Overall, the participants responded that $63.9 \%$ of the decisions they made during routine medical practice were typically the result of shared decision-making. For first- and secondyear residents, the reported rate was $63.5 \%$, and for third- and fourth-year residents, the rate was $65.1 \%$. A total of $65.8 \%$ of professors responded that they engaged in shared decisionmaking during their practice; however, only $50.0 \%$ of fellows answered the same way, which was lower than the percentage reported by other medical personnel.

\section{Shared decision-making during the process of withholding or withdrawing life-sustaining treatment}

For the item that asked whether the respondents' most recent decision to withhold or withdraw life-sustaining treatment was the result of shared decision-making, $88.6 \%$ of the 202 participants reported that the decision was shared. In total, 135 of the 179 participants provided the reason that they believed the decision was shared (Figure 1A). The most common reasons were that, from the doctor's perspective, a sufficient explanation was provided to the patient and his or her caregivers regarding the patient's current condition and prognosis, an autonomous decision was made that reflected the values of patients and caregivers, and sufficient time was provided for patients and caregivers to make a decision.

Among the 23 participants who responded they did not en- 


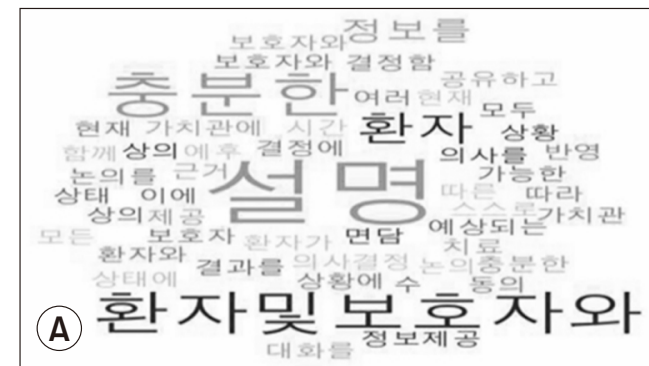

Reason to think it was a shared decision-making process

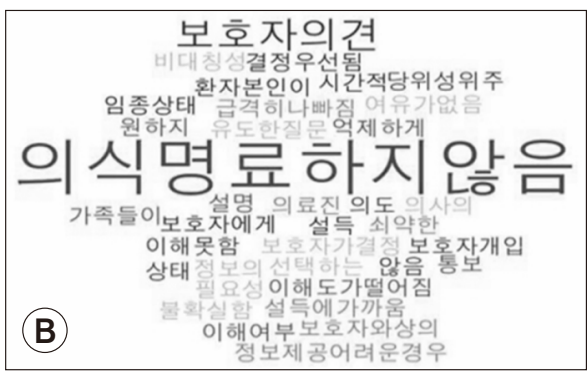

Reason to think it wasn't a shared decision-making process
Figure 1. Word cloud on the reasons for implementing shared decision making.
Table 2. Expected Satisfaction of Patients, Doctors, and Guardians with LifeSustaining Care Decisions ( $\mathrm{N}=201)$.

\begin{tabular}{lccccccccc}
\hline & \multicolumn{2}{c}{ Patient } & & \multicolumn{2}{c}{ Caregiver } & & \multicolumn{2}{c}{ Doctor } \\
\cline { 2 - 3 } \cline { 8 - 9 } \cline { 7 - 9 } & $\mathrm{n}$ & $\%$ & & $\mathrm{n}$ & $\%$ & & $\mathrm{n}$ & $\%$ \\
\hline Dissatisfied & 28 & 13.9 & & 17 & 8.4 & & 28 & 13.9 \\
Neutral & 90 & 44.6 & & 68 & 33.7 & & 73 & 36.1 \\
Satisfied & 83 & 41.1 & & 116 & 57.4 & & 100 & 49.5 \\
\hline
\end{tabular}

gage in shared decision-making, 21 provided reasons why (Figure 1B). Their answers included that decisions were made by caretakers while patients were not fully conscious after a rapid decline in their health status, doctors asked leading questions, and patients and caregivers did not have sufficient information due to an asymmetry in their understanding of medical information.

\section{Patients', caregivers', and doctors' satisfaction during the process of decision-making about withholding/withdrawing life-sustaining treatment}

The highest proportion of positive responses regarding the expected satisfaction of doctors, patients, and caregivers during the shared decision-making process related to the withdrawal of life-sustaining treatment was for caregivers at $57.4 \%$, followed by doctors at $49.5 \%$, and patients at $41.1 \%$ (Table 2). The participants responded that $13.9 \%$ of patients and doctors and $8.4 \%$ of caregivers were likely to be unsatisfied.

\section{Appropriateness of the shared decision-making process related to withholding/withdrawing life- sustaining treatment}

Participants were asked whether shared decision-making was conducted appropriately during the process of withholding/ withdrawing life-sustaining treatment, and $38.1 \%$ of respon-
Table 3. Beliefs Regarding Whether Shared Decision-Making was Appropriate for Making Life-Sustaining Treatment Decisions ( $\mathrm{N}=202)$.

\begin{tabular}{lccc}
\hline & \multicolumn{2}{c}{ Was SDM appropriate? } & \\
\cline { 2 - 3 } \multicolumn{1}{c}{ Variables } & $\begin{array}{c}\text { Yes } \\
(\mathrm{n}=77)\end{array}$ & $\begin{array}{c}\text { No or unknown } \\
(\mathrm{n}=125)\end{array}$ & \\
\hline Gender & & & 0.664 \\
Male & $37(48.1 \%)$ & $65(52.0 \%)$ & \\
Female & $40(51.9 \%)$ & $60(48.0 \%)$ & \\
Age (yr) & & & 0.822 \\
$\leq 40$ & $69(89.6 \%)$ & $110(88.0 \%)$ & \\
41 65 & $8(10.4 \%)$ & $15(12.0 \%)$ & \\
Position & & & 0.048 \\
Internal medicine resident & $66(85.7 \%)$ & $93(74.4 \%)$ & \\
Hematologist/ & $10(14.3 \%)$ & $31(25.6 \%)$ & \\
$\quad$ medical oncologist & & & \\
Type of institution & & & \\
Tertiary referral hospital & $60(77.9 \%)$ & $93(74.4 \%)$ & \\
General hospital & $17(22.1 \%)$ & $32(25.6 \%)$ & \\
Average number of patients per week & & 0.372 \\
$<100$ & $64(83.1 \%)$ & $96(76.8 \%)$ & \\
$\geq 100$ & $13(16.9 \%)$ & $29(23.2 \%)$ & \\
Education on SDM & & & \\
Received & $21(27.6 \%)$ & $26(20.8 \%)$ & \\
Not received or unknown & $55(72.4 \%)$ & $99(79.2 \%)$ & \\
\hline
\end{tabular}

SDM: shared decision making.

dents answering positively. However, $61.9 \%$ of the participants responded negatively or were uncertain. There were no differences according to sex, age, hospital tier, the number of patients treated per week, and whether the participant had received education about shared decision-making between those who responded that the shared decision-making process was appropriate and those who responded that it was not (Table 3). However, a higher proportion of residents than specialists responded that shared decision-making was practiced appropriately $(\mathrm{P}=0.048)$. The most frequent answer to the reason why shared decision-making was not carried out adequately during the process of withholding/withdrawing life-sustaining 
Table 4. Obstacles to the Use of Shared Decision-Making in Life-Sustaining Care Decisions ( $N=125)$.

\begin{tabular}{|c|c|c|c|}
\hline Variables & Resident ( $\mathrm{n}=93)$ & $\begin{array}{l}\text { Hematologist/ } \\
\text { medical oncologist } \\
\quad(n=32)\end{array}$ & P-value \\
\hline 1) Time pressure & $81(87.1 \%)$ & $31(96.9 \%)$ & 0.181 \\
\hline 2) Fee-for-service & $13(14.0 \%)$ & $5(15.6 \%)$ & 0.778 \\
\hline 3) Request of caregivers: unrealistic expectations, etc. & $58(62.4 \%)$ & $20(62.5 \%)$ & 1.000 \\
\hline 4) Ambiguity in the timing of the decision concerning LST & $52(55.9 \%)$ & $15(46.9 \%)$ & 0.416 \\
\hline $\begin{array}{l}\text { 5) Failure to provide adequate information about the patient's disease status prior to } \\
\text { making a decision concerning LST }\end{array}$ & $36(38.7 \%)$ & $14(43.8 \%)$ & 0.678 \\
\hline 6) Differences in patients' preferences for participation in decisions concerning LST & $20(21.5 \%)$ & $11(31.4 \%)$ & 0.160 \\
\hline 7) Complex medical terms and information & $26(28.0 \%)$ & $5(15.6 \%)$ & 0.235 \\
\hline 8) Patients/caregivers prefer doctors to make a decision concerning LST & $38(40.9 \%)$ & $13(40.6 \%)$ & 1.000 \\
\hline 9) Not feeling the need to practice shared-decision making & $7(7.5 \%)$ & $1(3.1 \%)$ & 0.679 \\
\hline 10) Lack the ability to practice shared-decision making & $10(10.8 \%)$ & $1(3.1 \%)$ & 0.287 \\
\hline 11) Not thinking that SDM will produce good results & $10(10.8 \%)$ & $2(6.3 \%)$ & 0.729 \\
\hline 12) No educational materials or tools to help with the decision-making process & $33(35.3 \%)$ & $13(40.6 \%)$ & 0.673 \\
\hline 13) Lack of education on how to make shared decisions & $22(23.7 \%)$ & $11(34.4 \%)$ & 0.252 \\
\hline
\end{tabular}

LST: life-sustaining treatment, SDM: shared decision-making.

treatment was time pressure $(89.6 \%, \mathrm{n}=112)$. There were no significant differences between the opinions of residents and specialists regarding the reason why appropriate shared decision-making does not occur (Table 4).

\section{DISCUSSION}

Given the reality of the clinical field in South Korea, the gap between the ideal and the reality related to doctor-patient shared decision-making during the process of withdrawing life-sustaining treatment is large. The reasons for this gap are that doctors lack understanding and rarely receive any training about shared decision-making. In addition, cancer patients and their caregivers often experience considerable anxiety about information provided to them during the shared decision-making process or experience difficulty accepting information in order to maintain hope. Lastly, a lack of time or issues with the health insurance system can make shared decision-making difficult to practice $[17,18]$. According to the results of this study, doctors often made decisions about withdrawing life-sustaining treatment without having received sufficient education about shared decision-making (76.2\%) and perceived this as a barrier to using practicing shared decision-making when making decisions about life-sustaining treatment (Table 4). Nonetheless, according to the results of the word cloud that visualized the opinions of doctors about the decision-making process (Figure 1), doctors expressed that sufficient time should be spent on explanations and that they must communicate with patients to adequately practice shared decision-making, indicating that they recognized what was required without having been educated on shared decisionmaking specifically. A lack of time and the requests of caregivers were identified as reasons why doctors found it difficult to practice shared decision-making when making decisions about life-sustaining treatment, which is a similar finding to the results of previous studies [17-19].

Efforts should be made to practice shared decision-making since, despite the aforementioned barriers, shared decisionmaking related to withdrawing life-sustaining treatment helps patients with chronic illnesses, including cancer, exercise selfdetermination and enables high-quality decision-making that respects the choices of patients [20]. Models outlining the shared decision-making process have been developed and implemented internationally, and the attitudes of medical personnel regarding shared decision-making have been examined [21,22]. However, no in-depth research on this topic has been conducted in South Korea. No shared decision-making model has been proposed or adapted for a South Korean context, and the attitudes and perceptions of doctors in South Korea regarding shared decision-making are unknown. The research 
team from the present study explored attitudes and perceptions about shared decision-making during end-of-life care among hematologists/medical oncologists and internal medicine residents who frequently participate in decisions about lifesustaining treatment in the clinical field after the enactment of the Act on Decisions on Life-Sustaining Treatment.

During a literature review of studies about doctors' perceptions and attitudes during shared decision-making, a 2015 study was found on the perceptions and attitudes about shared decision-making among American emergency medicine doctors in which $58 \%$ of respondents stated that shared decisionmaking is necessary, should be practiced when selecting treatment, and can prevent overtreatment [23]. The barriers to practicing shared decision-making according to the study were patients wanting doctors to make decisions, patients selecting more aggressive treatment methods when given the choice, and the complicated process of allowing patients to make choices about treatment. In a study that compared knowledge and attitudes about shared decision-making among surgeons, general practitioners, physician assistants, and nurse assistants, $84.2 \%$ of the 272 participants, of whom 100 were doctors, responded that shared decision-making was consistent with clinical guidelines. Seventy-five percent of the participants had a positive attitude about shared decision-making, responding that practicing shared decision-making does not take a significant amount of time [16].

In another study, Jo et al. [24] analyzed the effects of the characteristics of medical personnel on shared decisionmaking during end-of-life care. Age, work experience, moral sensitivity, and attitudes about dignified death were found to be associated with practicing shared decision-making. Among the participants' characteristics, moral sensitivity and a positive attitude toward dignified death were important factors that influenced shared decision-making. In the study by Jo et al., moral sensitivity and a positive attitude toward dignified death were higher for participants with more work experience and who were older. In this study, specialists $(n=11,25.6 \%) \mathrm{re}^{-}$ ported a lower percentage of adequate shared decision-making when making decisions about life-sustaining treatment than residents ( $n=66,41.5 \%$ ), which contradicts the previous study by Jo et al. The main reason for this finding could be time constraints, since specialists tend to have a higher workload than residents and may not have enough time to adequately participate in decision-making. Overall, $75.7 \%$ of hematologists/medical oncologists worked at tertiary hospitals. It can be assumed that a busy clinical schedule made it difficult to allocate sufficient time for the degree of communication needed for shared decision-making.

In addition, specialists may have a better understanding of the differences in patients' preferences related to discussions about life-sustaining treatments, since patients often delegate decisions related to life-sustaining treatment to family members or attending physicians who specialize in hematology and oncology. In contrast, a study of Swiss doctors found that the individual characteristics of doctors influenced the decisionmaking process related to withdrawing life-sustaining treatment. In the Swiss study, the most significant indicators after controlling for other factors such as patient characteristics were identified. After analyzing shared decision-making related to 2,542 deaths, the gender and religion of doctors were found not to be significant, while those who graduated after 2000 (relative risk ratio $=1.73,95 \%$ confidence interval $=1.27 \sim 2.37$ ) were more likely to practice shared decision-making than doctors who graduated before 2000 [25]. One reason for this could be that doctors who graduated earlier were not taught about shared decision-making; however, this study could not confirm any association between shared decision-making and education related to shared decision-making.

This study attempted to examine the satisfaction of $\mathrm{pa}^{-}$ tients and caregivers according to doctors related to decisionmaking about life-sustaining treatment and the factors that influenced satisfaction, but no significant results were found. In the future, it could be more meaningful to study consistency concerning decision-making between doctors and patients or caregivers and their satisfaction to understand any gaps. For this proposed study, the Shared Decision Making Questionnaire (SDM-Q), a measurement tool about shared decisionmaking from the perspective of doctors and patients, can be used [26]. This tool measures the degree to which shared decision-making is practiced according to patients (SDMQ-9) and doctors (SDM-Q-Doc) using 9 items. This tool has been used in studies to compare respondents' scores before and after they attend shared decision-making interventions to examine consistency regarding shared decision-making from 
the perspective of doctors and patients [27]. The tool has also been translated into Korean, though it has not been used to study the beliefs of cancer patients regarding decisions about life-sustaining treatments in South Korea; therefore, studies should be conducted first. The authors of the present study have also conducted a pilot study to examine the degree of shared decision-making practiced with 21 progressive cancer patients and patients with chronic renal failure scheduled for dialysis and their attending physicians (5 hematology and oncology specialists and nephrologists) using the SDM-Q-9 and SDM-Q-Doc tools (unpublished data). Valid results were not extracted from this study since the patients overestimated the degree of shared decision-making and the sample size was too small.

There are several limitations to this study. First, the selfevaluations and attitudes related to shared decision-making when making decisions about life-sustaining treatments among all internal medicine and hematology and oncology specialists cannot be known since only a subset of teaching hospitals that treat cancer patients in South Korea participated. Moreover, the attitudes regarding shared decision-making of doctors in other departments who treat cancer patients could not be confirmed. Second, while the validity of the survey was ensured by selecting survey items that were used in another study and finalizing them via an expert review, the reliability of the items was not tested. Follow-up studies should therefore test the reliability of the items. Third, since only doctors' subjective beliefs and attitudes concerning shared decision-making about life-sustaining treatments were included, it was not possible to compare the beliefs and attitudes of doctors with those of patients and caregivers. This limitation can be addressed in the aforementioned proposed study using the SDM-Q. However, as mentioned, further consideration of the methodology when using the SDM-Q as the tool for measuring shared decisionmaking about life-sustaining treatment is needed.

This is the first study in South Korea to examine the selfevaluations and beliefs of doctors related to shared decisionmaking, who now play an even more important role in withholding or withdrawing life-sustaining treatment during endof-life care following the enactment of the Act on Decisions on Life-sustaining Treatment. Most participants (88.6\%) responded that they practiced shared decision-making when making decisions about life-sustaining treatment. However, only $38.1 \%$ responded that appropriate shared decision-making was typically practiced. Barriers must still be overcome, the most significant of which was the lack of time to practice shared decision-making.

Sufficient communication is very important during endof-life care. Public policy should be improved to resolve the issue of lack of time for treatment. Education about shared decision-making methods should also be provided for doctors. The authors plan to analyze data from a larger sample of doctors in collaboration with the researchers of a shared decisionmaking study conducted by the Korean Society of Nephrology and to further explore the reality of shared decision-making in the South Korean clinical field. Through these efforts, a shared decision-making model that reflects various clinical situations including end-of-life care should be developed for South Korea, and follow-up studies are needed that provide data to support relevant policy.

\section{CONFLICT OF INTEREST}

No potential conflict of interest relevant to this article was reported.

\section{ORCID}

Dalyong Kim, https://orcid.org/0000-0003-0193-422X Hyun Jung Lee, https://orcid.org/0000-0001-8499-736X Soo-Young Yu, https://orcid.org/0000-0003-0862-7687 Jung Hye Kwon, https://orcid.org/0000-0002-5965-3204 Hee Kyung Ahn, https://orcid.org/0000-0001-7786-7233 Jee Hyun Kim, https://orcid.org/0000-0003-1336-3620 Seyoung Seo, https://orcid.org/0000-0003-1201-3194 Chi Hoon Maeng, https://orcid.org/0000-0002-4450-3350 Seungtaek Lim, https://orcid.org/0000-0001-7258-2263 Do Yeun Kim, https://orcid.org/0000-0001-7265-1994 Sung Joon Shin, https://orcid.org/0000-0002-0777-9278 


\section{AUTHOR'S CONTRIBUTIONS}

Conception or design of the work: HJL, JHK, HKA, JHK, SS, CHM, SL, DYK, SJS. Data collection: HJL, JHK, HKA, JHK, SS, CHM, SL. Data analysis and interpretation: DK, SYY. Drafting the article: DK. Critical revision of the article: DYK, SJS. Final approval of the version to be published: all authors.

\section{SUPPLEMENTARY MATERIALS}

Supplementary materials can be found via https://doi. org/10.14475/jhpc.2021.24.4.204.

\section{REFERENCES}

1. Sleeman KE, Collis E. Caring for a dying patient in hospital. BMJ 2013;346:f2174.

2. Charles C, Gafni A, Whelan T. Shared decision-making in the medical encounter: what does it mean? (or it takes at least two to tango). Soc Sci Med 1997;44:681-92.

3. Muller-Engelmann M, Donner-Banzhoff N, Keller H, Rosinger L, Sauer C, Rehfeldt K, et al. When decisions should be shared: a study of social norms in medical decision making using a factorial survey approach. Med Decis Making 2013;33:37-47.

4. The National Law Information Center. Act on decisions on life-sustaining treatment for patients in hospice and palliative care or at the end of life 2016 [Internet]. Sejong: Ministry of Government Legislation; 2016 [cited 2021 Oct 5]. Available from: https://www.law.go.kr/LSW/eng/ engLsSc.do? menuld=2\&section=lawNm\&query=sustaining \& $\mathrm{x}=0$ \&y=0\#liBgcolor0.

5. Yoo SH, Choi W, Kim Y, Kim MS, Park HY, Keam B, et al. Difficulties doctors experience during life-sustaining treatment discussion after enactment of the life-sustaining treatment decisions act: a cross-sectional study. Cancer Res Treat 2021;53:584-92.

6. Sabatino CP. The evolution of health care advance planning law and policy. Milbank Q 2010;88:211-39.

7. Belanger E, Rodriguez C, Groleau D. Shared decision-making in palliative care: a systematic mixed studies review using narrative synthesis. Palliat Med 2011;25:242-61.

8. Padilla Garrido N, Aguado Correa F, Bayo Lozano E, Bayo Calero J, Ortega Moreno M. Physicians' awareness and assessment of shared decision making in oncology practice. Rev Esp Salud Publica 2019;93:e201910066.

9. Korean Society of Critical Care Medicine. Korean professional consensus for comfort care and withdrawing/withholding in the intensive care unit; by the task force of Korean Society of Critical Care Medicine. Seoul:KSCCM;2018.

10. Jo KH. Development and evaluation of shared medical decision-making scale for rnd-of-life patients in Korea. J Korean Acad Nurs 2012;42:453-65.

11. Lee $N-Y$, Lee S, Lee S-K. The perceptions and needs of hospice palliative care and shared decision making among middle-aged adults. Korean J Hosp Palliat Care 2016;19:310-21.

12. docdocdoc.co.kr [Internet]. Seoul: docdocdoc.co.kr: 2019 [cited 2021 Oct 5]. Available from: http://www.docdocdoc.co.kr/news/articleView.html? idxno=1070270.

13. Kanzaria HK, Brook RH, Probst MA, Harris D, Berry SH, Hoffman JR. Emergency physician perceptions of shared decision-making. Acad Emerg Med 2015;22:399-405.

14. Tamirisa NP, Goodwin JS, Kandalam A, Linder SK, Weller S, Turrubiate S, et al. Patient and physician views of shared decision making in cancer. Health Expect 2017;20:1248-53.

15. Rusiecki J, Schell J, Rothenberger S, Merriam S, McNeil M, Spagnoletti C. An innovative shared decision-making curriculum for internal medicine residents: findings from the University of Pittsburgh Medical Center. Acad Med 2018;93:937-42.

16. Forcino RC, Yen RW, Aboumrad M, Barr PJ, Schubbe D, Elwyn G, et al. US-based cross-sectional survey of clinicians' knowledge and attitudes about shared decision-making across healthcare professions and specialties. BMJ Open 2018;8:e022730.

17. Lee HR, Lim C, Yun HG, Kang SH, Kim DY. Making an informed decision of Korean cancer patients: the discrepancy between a patient's recall of information and the information needed for acquisition of radiotherapy informed consent. Supportive Care Cancer 2018;26:297-303.

18. Bae J-M. Shared decision making: relevant concepts and facilitating strategies. Epidemiol Health 2017;39:e2017048.

19. Visser M, Deliens L, Houttekier D. Physician-related barriers to communication and patient- and family-centred decision-making towards the end of life in intensive care: a systematic review. Crit Care 2014;18:604.

20. AHRQ. The SHARE approach: a model for shared decisionmaking - fact sheet. Rockville, MD: Agency for Healthcare Research and Quality; 2016. Available from: https://www.ahrq.gov/health-literacy/professional-training/shared-decision/tools/factsheet.html.

21. Elwyn G, Frosch D, Thomson R, Joseph-Williams N, Lloyd A, Kinnersley P, et al. Shared decision making: a model for clinical practice. J Gen Intern Med 2012;27:1361-7.

22. Ho A, Jameson K, Eiser A. Sowing the SEED for patient empowerment. Am J Bioeth 2017;17:42-5. 
23. Kanzaria HK, Brook RH, Probst MA, Harris D, Berry SH, Hoffman JR. Emergency physician perceptions of shared decision-making. Acad Emerg Med 2015;22:399-405.

24. Jo K-H, An G-J, Lee HS. Health care professional factors influencing shared medical decision making in Korea. SAGE Open 2015;5:2158244015614608.

25. Bopp M, Penders YW, Hurst SA, Bosshard G, Puhan MA, Group SE-0-LDS. Physician-related determinants of medical end-of-life decisions - A mortality follow-back study in Switzerland. PLoS one 2018;13:e0203960.

26. patient-als-partner.de [Internet]. SDM-Q-9/SDM-Q-Doc. Hamburg: Patient-als-Partner; 2019 [cited 2021 Oct 5]. Available from: http:// www.patient-als-partner.de/index.php?article_id=20\&clang=2/.

27. Doherr H, Christalle E, Kriston L, Harter M, Scholl I. Use of the 9-item Shared Decision Making Questionnaire (SDM-Q-9 and SDM-Q-Doc) in intervention studies-a systematic review. PLoS one 2017;12:e0173904. 